Artículo de Investigación

\title{
Evaluación de la concentración y distribución espacial de material particulado en los campus de la UNACH - Riobamba
}

\section{Evaluation of the concentration and spatial distribution of particulate material in the UNACH - Riobamba campuses}

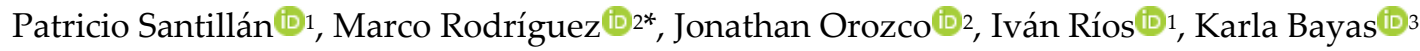 \\ ${ }^{1}$ Carrera de Ingeniería Ambiental, Facultad de Ingeniería, Universidad Nacional de Chimborazo, Riobamba, Ecuador, 060150; \\ psantillan@unach.edu.ec;irios@unach.edu.ec \\ 2Direccion de Investigación, Universidad Nacional de Chimborazo, Riobamba, Ecuador, 060150; jonathan.orozco@unach.edu.ec \\ ${ }^{3}$ Gobierno Autónomo Descentralizado del Cantón Alausí, Alausí, Ecuador, 060250; andreabayas12@hotmail.com \\ *Correspondencia: mvrodriguez@unach.edu.ec
}

\begin{abstract}
Citación: Santillán, P., Rodríguez, M. Orozco, J., Ríos, I., \& Bayas, K., (2021). Evaluación de la concentración y distribución espacial de material particulado en los campus de la UNACH - Riobamba. Novasinergia. 4(2). 111-126. https://doi.org/10.37135/ns.01.08.07
\end{abstract}

Recibido: 09 febrero 2021

Aceptado: 28 junio 2021

Publicado: 01 diciembre 2021

Novasinergia

ISSN: 2631-2654
Resumen: El objetivo de la presente investigación fue realizar un análisis comparativo de los niveles de concentraciones de Material Particulado Volátil y Sedimentable (PMV y PMS), en los tres campus de la Universidad Nacional de Chimborazo. El PMV se midió con el equipo DustTrak ${ }^{\mathrm{TM}}$ II por 24 días y el PMS se determinó por medio de un sistema de monitoreo pasivo. La caracterización de metales asociados al PM fue mediante espectroscopia de fotones rayos $\mathrm{X}$ dispersados (EDX). De acuerdo a la legislación ecuatoriana y la OMS, las concentraciones de $\mathrm{PM}_{2.5}$ en las estaciones de monitoreo no exceden los limites máximo permisibles. El PM10 presento un valor medio de $10 \mu \mathrm{g} / \mathrm{m}^{3}$, máximo de $15 \mu \mathrm{g} / \mathrm{m}^{3}$ y un mínimo de $6 \mu \mathrm{g} / \mathrm{m}^{3}$. Los metales asociados al PM son 11 elementos de origen antropogénico y natural, donde la principal fuente responsable es el tráfico, industria, construcción, partículas de polvo y ceniza. La distribución espacial del PM, determinó que los focos de emisión son de escala local; con un radio crítico de arrastre y deposición de $50 \mathrm{~m}$. Con ello se demuestra que la modelación de PM, a través de herramientas SIG, brinda resultados favorables para estudiar el comportamiento de las partículas.

Palabras clave: Calidad del aire, contaminación atmosférica, kriging, material particulado, material particulado sedimentable, material particulado volátil, $\mathrm{PM}_{2.5}, \mathrm{PM}_{10}$

\begin{abstract}
The objective of this research was to carry out a comparative analysis of the levels of concentrations of Volatile and Sedimentable Particulate Material (PMV and PMS), in the three campuses of the National University of Chimborazo. PMV was measured with the DustTrak ${ }^{\mathrm{TM}}$ II equipment for 24 days and PMS was determined through a passive monitoring system. The characterization of metals associated with PM was by scattered X-ray photon spectroscopy (EDX). According to the Ecuadorian legislation and the WHO, the concentrations of PM2.5 in the monitoring stations do not exceed the maximum permissible limits. PM1o presented a mean value of $10 \mu \mathrm{g} / \mathrm{m}^{3}$, a maximum of $15 \mu \mathrm{g} / \mathrm{m}^{3}$ and a minimum of $6 \mu \mathrm{g} / \mathrm{m}^{3}$. The metals associated with PM are 11 elements of anthropogenic and natural origin, where the main responsible source is traffic, industry, construction, dust particles and ash. The spatial distribution of PM determined that the emission sources are on a local scale; with a critical radius of drag and deposition of $50 \mathrm{~m}$. This shows that PM modeling, through GIS tools, provides favorable results to study the behavior of particles.
\end{abstract}

Keywords: Air quality, atmospheric pollution, kriging, particulate material, particulate material volatile, particulate material sedimentable, $P M_{2.5}, P M_{10}$ 


\section{Introducción}

La contaminación atmosférica por material particulado (PM), es uno de los mayores problemas en la actualidad (Santillán et al., 2016). Se presenta en todos los lugares, especialmente en sitios urbanos con mayor actividad industrial y donde se conjugan el transporte vehicular (Celis et al., 2007). Esta última se ha convertido en un fenómeno de constante crecimiento, ocasionando una amenaza para el medio ambiente, así como para la salud y la calidad de vida de sus habitantes (Palacio et al., 2014).

El PM atmosférico y sedimentable ocasiona daños a la salud y su peligrosidad va a depender de la concentración, tamaño y persistencia en el aire (Ubilla \& Yohannessen, 2017). Cabe indicar también que el PM $2.5 \mu \mathrm{m}$ y $10 \mu \mathrm{m}$ son partículas que al ingresar a la caja torácica causan efectos agudos y crónicos en la salud de las personas (Salinas, 2012). Por ello vale la pena mostrar que más del $80 \%$ de la población en países en vías de desarrollo están expuestos a niveles peligrosos de calidad del aire, que con frecuencia los contaminantes exceden los límites máximos permisibles (Hinojosa et al., 2019).

De acuerdo con la Organización Mundial de la Salud (OMS), la contaminación atmosférica a nivel mundial causa aproximadamente 4.2 millones de muertes prematuras al año (Scapini et al., 2018). La contaminación por PM es un problema a nivel global, se ha convertido en una amenaza tanto para la salud humana como para el ambiente; debido a que ocasiona enfermedades respiratorias (Ubilla \& Yohannessen, 2017). Es decir la incesante exposición a grandes concentraciones de PM, puede agravar enfermedades crónicas, cardiacas y pulmonares (Canales et al., 2014; Ubilla \& Yohannessen, 2017). Es por ello que se debe monitorear permanentemente y aplicar políticas en materia ambiental y de salud en todos los ámbitos desde locales, nacionales y mundiales para prever consecuencias (García \& Rojas, 2016).

La ciudad de Riobamba durante los últimos años ha tenido un crecimiento poblacional, ha pasado de tener 193.315 habitantes en el año 2001 a 225.741 habitantes en el 2010 (INEC, 2010). Este crecimiento poblacional se orienta al desarrollo acelerado de la urbe producto de la nueva ruralidad, donde un gran número de familias deciden migrar de la zona rural a la ciudad a fin de cambiar su actividad económica y mejorar sus ingresos que permiten obtener nuevos bienes y servicios (GADM Riobamba, 2020).

Este crecimiento poblacional en especial el parque automotor y la actividad industrial generan un grave problema de contaminación atmosférica. Además, la ciudad por ubicarse en la parte central de la cordillera andina está rodeada de volcanes, uno de ellos es el volcán Tungurahua que desde la época colonial, ha tenido varios períodos de actividad eruptiva entre: 1640 - 1641, 1773 - 777, 1886 1888, 1916 - 1918 y desde 1999 hasta el 2016 (Few et al., 2017). Este fenómeno natural está ligado con la contaminación de PM, en especial la ceniza volcánica que tiene un tamaño inferior a $2 \mathrm{~mm}$, lo que afecta directamente a la población y es fácilmente perceptible en las personas, convirtiéndose en una de las principales causas de infecciones respiratorias (Bustillos et al., 2018).

El objetivo de la presente investigación es determinar la concentración de PMV y PMS en los campus de la Universidad Nacional de Chimborazo, región sierra centro del Ecuador, mediante el método fotométrico utilizando el DustTrack II y método pasivo con el establecimiento de estaciones de monitoreo implementadas en sectores con mayor incidencia de vehicular. Las partículas de PM sedimentable fueron analizadas mediante el microscopio electrónico de barrido y la espectroscopia de energía de fotones dispersas. 


\section{Metodología}

\subsection{Sitios de muestreo}

El presente trabajo de investigación se realizó en los tres campus de la Universidad Nacional de Chimborazo (Unach), de la ciudad de Riobamba, en la región sierra centro del Ecuador, a una altitud de 2850 m.s.n.m. Según datos obtenidos de los anuarios del Instituto Nacional de Meteorología e Hidrología del Ecuador la temperatura promedio en la ciudad de Riobamba varía entre 9 y $21^{\circ} \mathrm{C}$; con una precipitación anual de $491 \mathrm{~mm}$ con rangos mensuales de (3-83 mm); una presión atmosférica promedio de $545 \mathrm{~mm}$ de $\mathrm{Hg}$ y una humedad relativa del 72\% (INAMHI, 2019).

En el área de estudio se colocaron ocho estaciones de monitoreo pasivo para determinar PMS y por un mes se monitoreó el PMV. La estación RF1 fue ubicada en el campus norte Edison Rivera (b); la RF2 en el campus centro (a) y la RF3 en el campus sur La Dolorosa (c) (Figura 1).

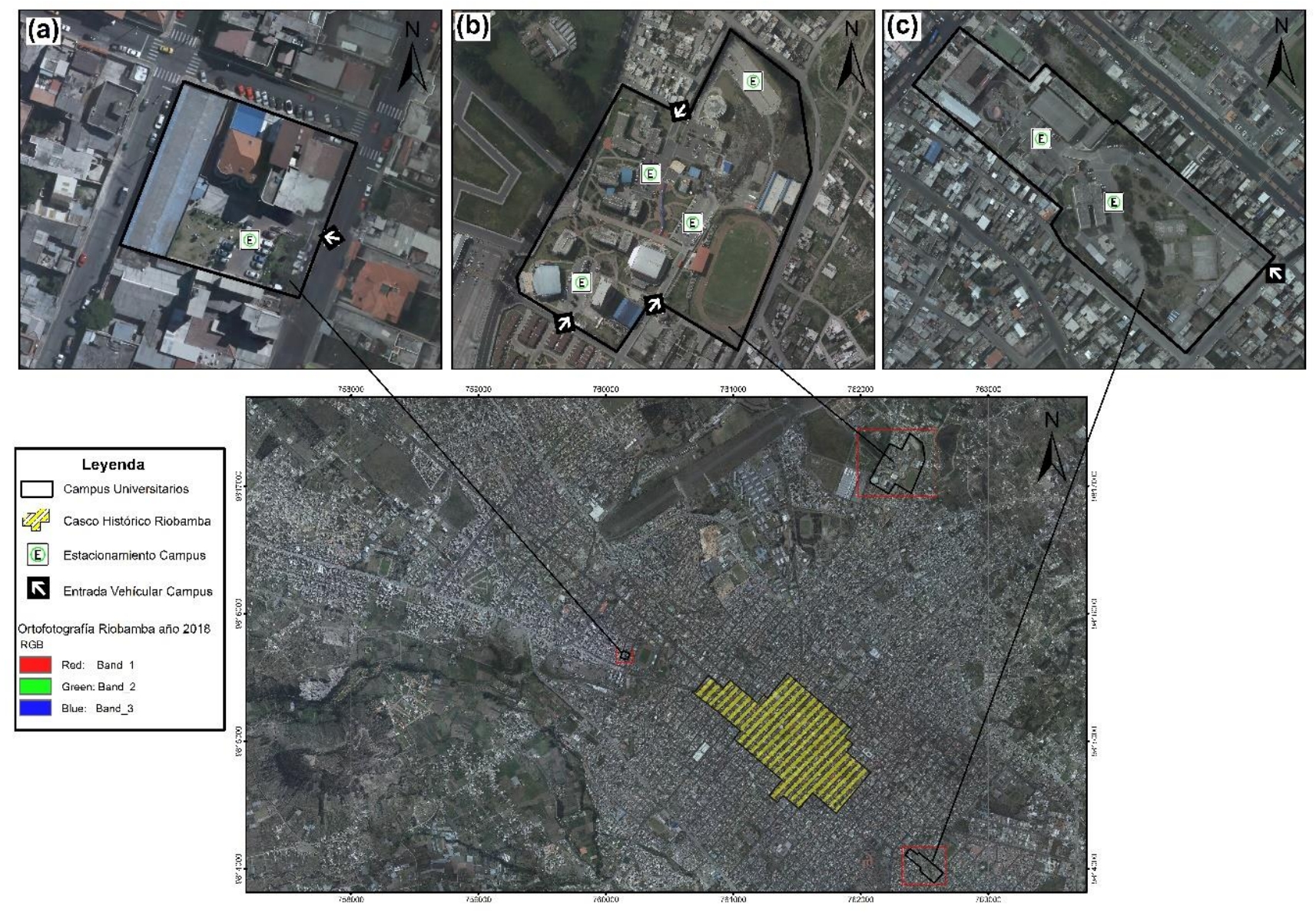

Figura 1: Área de estudio. (a) Campus: Centro; (b) Campus: Edison Riera y (c) Campus: La Dolorosa.

\subsection{Método de muestreo}

Para el muestreo de PMS se instalaron 3 puntos por área de estudio; la muestra fue recolectada por un periodo de 30 días de forma continua como se establece en el Libro VI Anexo IV del TULSMA; con el propósito de evaluar la concentración de partículas sedimentables en los sitios de monitoreo (Tabla 1). 
Tabla 1: Localización de los puntos de muestreo.

\begin{tabular}{|c|c|c|}
\hline Estaciones de muestreo & Localización & Observaciones \\
\hline \multirow{3}{*}{ Campus Norte Edison Rivera: RF1 } & 1. S: $1^{\circ} 39^{\prime} 7.8^{\prime \prime} \quad$ O: $78^{\circ} 38^{\prime} 33.7^{\prime \prime}$ & \multirow{3}{*}{ Alto tráfico vehicular } \\
\hline & 2. S: $1^{\circ} 39^{\prime} 6.2^{\prime \prime} \quad$ O: $78^{\circ} 38^{\prime} 32.3^{\prime \prime}$ & \\
\hline & 3. S: $1^{\circ} 39^{\prime} 14.7^{\prime \prime}$ O: $78^{\circ} 38^{\prime} 37.7^{\prime \prime}$ & \\
\hline \multirow{3}{*}{ Campus Centro: RF2 } & 1. S: 1³9'58.9" O: 78³9'41.9" & \multirow{3}{*}{$\begin{array}{l}\text { Medio tráfico } \\
\text { vehicular }\end{array}$} \\
\hline & 2. S: 1³9'59.4" O: 78³9'42.3" & \\
\hline & 3. S: 1³9'58.5" O: 78³9'42.7" & \\
\hline \multirow{3}{*}{ Campus Sur La Dolorosa: RF3 } & 1. S: $1^{\circ} 40^{\prime} 50.4^{\prime \prime} \mathrm{O}: 78^{\circ} 38^{\prime} 27.9^{\prime \prime}$ & \multirow{3}{*}{ Alto tráfico vehicular } \\
\hline & 2. S: $1^{\circ} 40^{\prime} 51.3^{\prime \prime} \mathrm{O}: 78^{\circ} 38^{\prime} 25.4^{\prime \prime}$ & \\
\hline & 3. S: $1^{\circ} 40^{\prime} 52.0^{\prime \prime} \mathrm{O}: 78^{\circ} 38^{\prime} 26.3^{\prime \prime}$ & \\
\hline
\end{tabular}

\subsection{Determinación de material particulado volátil}

Para el análisis de PMV se utilizó el equipo DustTrak ${ }^{\mathrm{TM}}$ II (TSI Incorporated/500 Cardigan Road/Shoreview, MN 55126/USA). Antes de iniciar con la medición el equipo fue previamente calibrado (ZERO CAL), con el filtro $0 \mu \mathrm{m}$ de elaboración del fabricante. Una vez calibrado el equipo se puso el filtro de $2.5 \mu \mathrm{m}$ y $10 \mu \mathrm{m}$ individualmente y se inició con la medición de PM. El análisis por muestra tarda 2 minutos aproximadamente y los valores son expresados en $\mathrm{mg} / \mathrm{m}^{3}$, que posteriormente son convertidos a $\mu \mathrm{m}$. Hay que destacar que las lecturas con el equipo DustTrak ${ }^{\mathrm{TM}}$ II, empiezan al momento de que el PM se dispersa por el aire. El tiempo de monitoreo fue de tres veces al día (8:00; 13:00 y 15:00), durante 24 días consecutivos.

\subsection{Determinación de material particulado sedimentable}

Para el análisis de PMS se empleó el método pasivo propuesto por Almirón et al., (2008). El cual propone colocar papel filtro en cajas Petri previamente secado, pesado y determinado su área. El tiempo establecido para el monitoreo es de 30 días. El resultado se alcanza mediante el cálculo de los pesos final e inicial y dividiendo para el área donde se recogió la muestra; obteniendo valores en unidades de $\mathrm{mg} / \mathrm{cm}^{2}$ durante 1 mes (Ecuación 1).

$$
P A S=\left(\frac{P f-P i}{\text { Área }}\right) 1 m e s
$$

Dónde:

PAS: Polvo Atmosférico Sedimentable

Pi: Peso del papel filtro después de salir de laboratorio

Pf: Peso del papel filtro después de estaré expuesto al periodo de muestreo

Área del papel filtro: $A=\pi \times r^{2}$ (Santillán et al., 2016).

\subsection{Análisis de microscopia electrónica de barrido de material particulado sedimentable}

Para comprender las composiciones elementales de partículas finas de PMS, se utilizó el microscopio electrónico de barrido (VEGA 3 SEM - EDX Tescam Company Alemania), el cual toma imágenes a 100x y 200x, 50x, 20x y 10x. El análisis de las imágenes se desarrolló con el software Essence ${ }^{T M}$ de TESCAN. Esta combinación simplifica significativamente la adquisición de datos morfológicos y elementales de la muestra, lo que convierte a VEGA SEM en una solución analítica eficiente para la inspección de materiales de rutina. Las muestras de papel filtro fueron cortadas de $1 \mathrm{~cm}$ y colocadas sobre el porta muestras de aluminio y recubiertas (metalizadas) con partículas de oro para obtener las mejores condiciones de imagen. Este pre tratamiento permite que la superficie 
de la muestra fuera conductora. Las observaciones se realizaron en un SEM - EDX modelo Essence ${ }^{\mathrm{TM}}$ 3D Collision, a un voltaje de aceleración de 5-10 kV. Las imágenes muestran la morfología, composición química, tamaño y distribución de las partículas. En las imágenes se puede observar la morfología, composición química, tamaño y distribución de las partículas presentes en las muestras recolectadas en los campus de la Unach.

\subsection{Modelo geoestadístico}

El análisis de distribución espacial se desarrolló con el software ArcGIS 10.x, el cual utiliza modelos de datos inteligentes que permiten representar variables continuas en el espacio. Este programa incluye varias herramientas; pero para nuestro análisis de interpolación de datos se utilizó las herramientas Spatial Analyst y Geostatistical Analyst. Para programar la interpolación se consideró valores de $\mathrm{PM}_{2.5}$, el cual fue obtenido mediante el muestreo durante 24 días consecutivos. Además, se utilizó valores de velocidad del viento que para la ciudad de Riobamba fue de $2.1 \mathrm{~m} / \mathrm{s}$ y dirección del viento en la mayoría de los casos sopla principalmente desde el norte hacia el sur (N-S).

\subsection{Análisis estadístico}

Se realizaron pruebas de normalidad para verificar los supuestos del análisis ANOVA agrupando los datos de PMV asociado a las fracciones de PM2.5 y PM10. Se realizó un ANOVA de una sola vía, $(\mathrm{p}<0,05)$, para detectar diferencias estadísticas entre tratamientos y pruebas de medias de Tukey. Para este análisis estadístico se utilizó el software MINITAB V17 (Minitab, 2016).

\section{Resultados}

\subsection{Material particulado volátil}

La concentración de $\mathrm{PM}_{2.5}$ y PM10, en las tres estaciones de monitoreo RF1, RF2, y RF3, establecidas en la Unach, se muestran en la figura 2. En el campus Edison Riera (RF1) la concentración diaria de $\mathrm{PM}_{2.5}$ vario de $8 \mu \mathrm{g} / \mathrm{m}^{3}$ a $74 \mu \mathrm{g} / \mathrm{m}^{3}$. En el campus Centro (RF2), los valores fluctuaron entre $7 \mu \mathrm{g} / \mathrm{m}^{3} \mathrm{a} 54 \mu \mathrm{g} / \mathrm{m}^{3}$ y en el campus la Dolorosa (RF3), la concentración diaria mostro valores que van desde $7 \mu \mathrm{g} / \mathrm{m}^{3}$ a $37 \mu \mathrm{g} / \mathrm{m}^{3}$. Estos valores no exceden los límites máximos permisibles establecidos por la legislación ecuatoriana $\left(65 \mu \mathrm{g} / \mathrm{m}^{3}\right)$ y la OMS $\left(25 \mu \mathrm{g} / \mathrm{m}^{3}\right)$.

Con respecto a la concentración de PM10, en la estación RF1, durante los 24 días de monitoreo, presento un valor medio de $10 \mu \mathrm{g} / \mathrm{m}^{3}$, con un valor máximo de $15 \mu \mathrm{g} / \mathrm{m}^{3}$ y un mínimo de $6 \mu \mathrm{g} / \mathrm{m}^{3}$; la estación RF2 el valor medio fue de $10 \mu \mathrm{g} / \mathrm{m}^{3}$, con un máximo de $14 \mu \mathrm{g} / \mathrm{m}^{3} \mathrm{y}$ un mínimo de $8 \mu \mathrm{g} / \mathrm{m}^{3}$ y en la estación RF3 mostro un valor medio de $10 \mu \mathrm{g} / \mathrm{m}^{3}$, con un valor máximo de $14 \mu \mathrm{g} \mathrm{m} \mathrm{m}^{3} \mathrm{y}$ un mínimo de $7 \mu \mathrm{g} / \mathrm{m}^{3}$. Sin embargo, en las estaciones RF1 y RF2, se obtuvieron valores máximos de $\mathrm{PM}_{2.5}\left(74 \mu \mathrm{g} / \mathrm{m}^{3}\right.$ y $\left.54 \mu \mathrm{g} / \mathrm{m}^{3}\right)$, los días 12 y 24 respectivamente. El valor registrado el día 12 fue el único que sobrepaso el límite permisible de acuerdo a la legislación ecuatoriana (Bayas, 2017). Mientras tanto el PM10 alcanzó su máximo valor el día 1 con $15 \mu \mathrm{g} / \mathrm{m}^{3}$ en la estación RF1 lo que obedece al alto tráfico vehicular característico del área en estudio y el mínimo valor se registró los días 20 y 24 con $6 \mu \mathrm{g} / \mathrm{m}^{3}$ cada uno. 


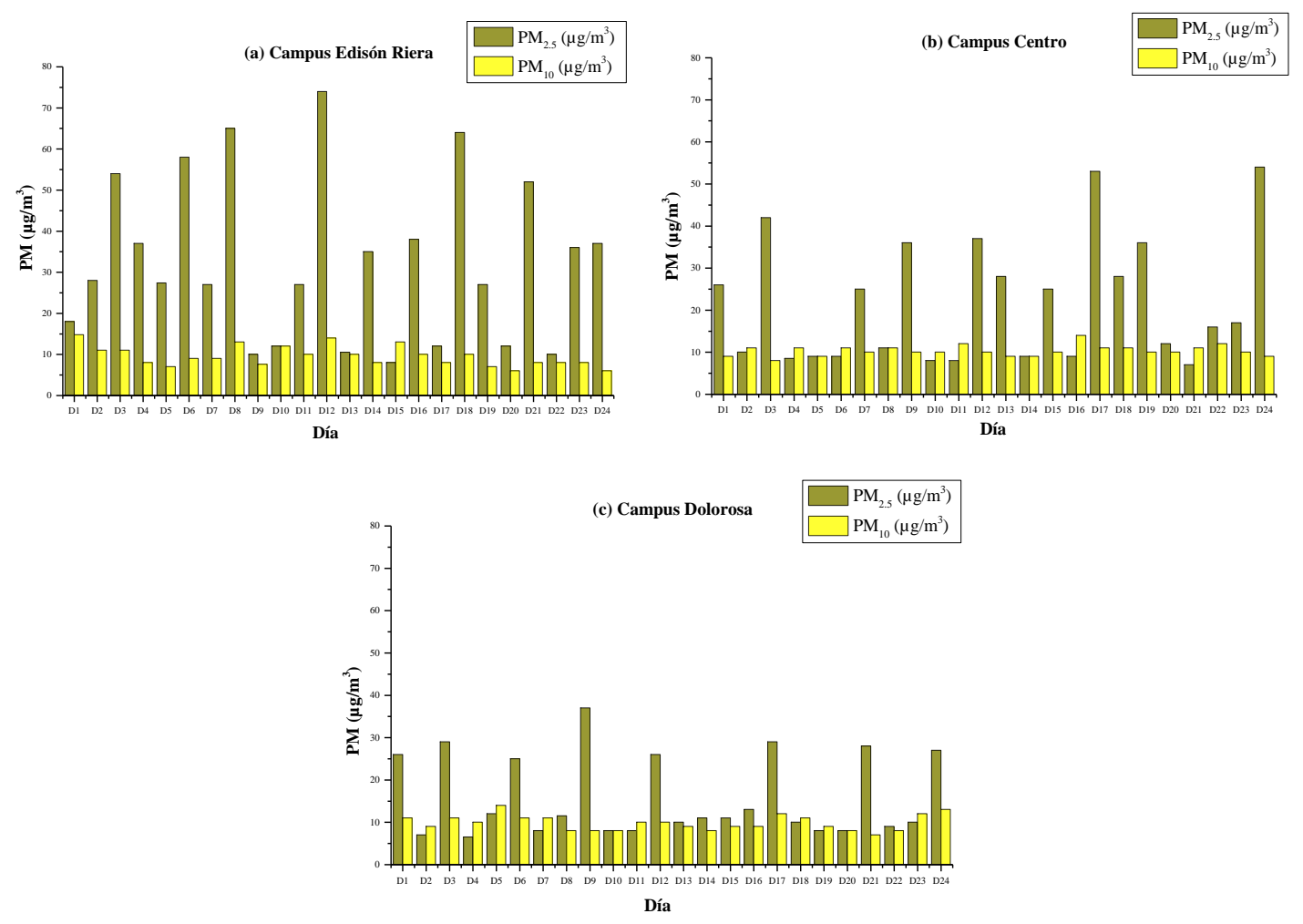

Figura 2: Concentración de PM en los campus de la Unach.

3.2. Comparación de material particulado volátil asociado a las fracciones de PM2.5 y PM10

Las concentraciones medias de $\mathrm{PM}_{2.5}$ registradas en el área de estudio, así como los valores de desviación estándar obtenidos para cada uno de los sitios se indican en la Tabla 2.

Tabla 2: Estadística descriptiva de PMV asociado a la fracción PM2.5.

$\begin{array}{cccccc}\text { Campus } & \text { Media } & \text { SD } & \text { Mínimo } & \text { Máximo } & \text { IC de } 95 \% \\ \text { RF1 } & 10.82 & 6.61 & 2.67 & 24.67 & (8.74 ; \\ \text { RF2 } & 5.25 & 3.16 & 2.17 & 12.33 & (3.17 ; 7.33) \\ \text { RF3 } & 7.27 & 4.92 & 2.33 & 18.00 & (5.20 ; 9.35)\end{array}$

En la tabla 3 se muestra la concentración media asociada a la fracción PM10. En el área de estudio podemos notar que uno de los factores que aporta al incremento de la contaminación atmosférica es la combustión del parque automotor que continuamente se incrementa y no recibe un adecuado mantenimiento. También la emisión de polvos de sectores aledaños son las principales fuentes de emisiones. Con ello el presente estudio proporciona información importante que puede ser utilizada para ayudar a las autoridades institucionales a tomar decisiones y definir planes locales para la gestión de la calidad del aire y para aumentar la conciencia de la población.

Tabla 3: Estadística descriptiva de PMV asociado a la fracción PM10.

\begin{tabular}{lccccc} 
Campus & Media & SD & Mínimo & Máximo & IC de 95\% \\
RF1 & 3.17 & 0.82 & 2.00 & 4.93 & $(2.91 ; 3.43)$ \\
RF2 & 3.28 & 0.60 & 2.33 & 4.67 & $(3.02 ; 3.54)$ \\
RF3 & 3.44 & 0.42 & 2.67 & 4.67 & $(3.18 ; 3.70)$ \\
\hline
\end{tabular}


La figura 3 muestra el valor medio e intervalo de confianza del 95\% para la concentración de PM2.5. encontradas a partir de las mediciones gravimétricas, en las áreas de estudio. Este valor corresponde a las tres mediciones diarias obtenidas con el equipo DustTrak ${ }^{\mathrm{TM}}$ II, donde se observa que las concentraciones medias de $\mathrm{PM}_{2.5}$ y $\mathrm{PM}_{10}$ obtenidas en los campus universitarios no evidencian cambios significativos (Figura 3). Los valores diarios obtenidos gravimétricamente para PM2.5. y PM10 estuvieron entre los 7 a $74 \mu \mathrm{g} / \mathrm{m}^{3}$ y 6 a $15 \mu \mathrm{g} / \mathrm{m}^{3}$ respectivamente. Estas diferencias pueden estar asociadas con el tipo y cantidad de tráfico vehicular que circula por las vías internas de los campus universitarios. Por lo que esta evidencia puede convertirse en un indicador de la importancia y el impacto que ocasionan las emisiones de los vehículos que circulan por el interior de la institución de educación superior.
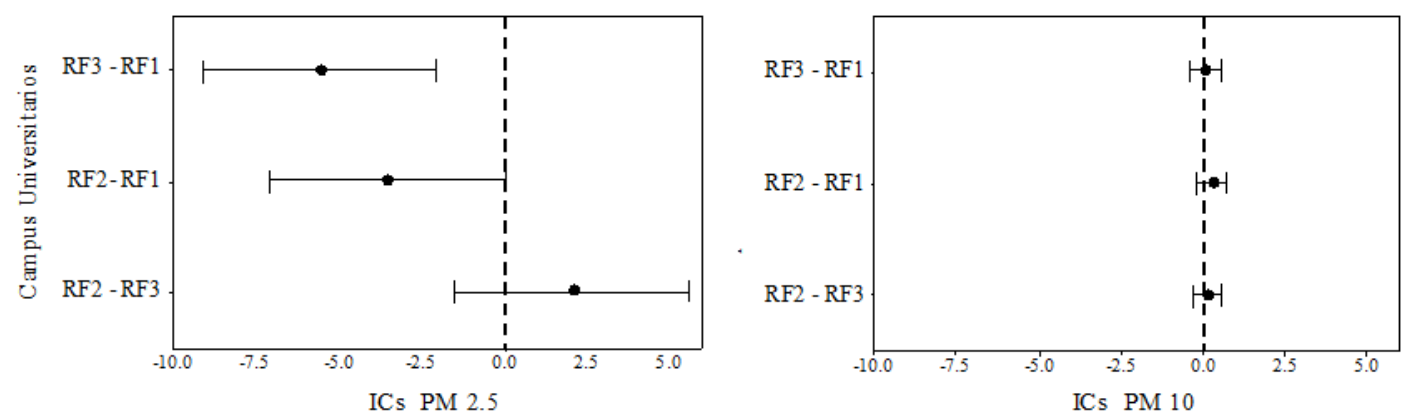

Figura 3: Media de intervalo de confianza del 95\% para las concentraciones de PM2.5 y PM10.

\subsection{Caracterización química}

El análisis de composición química mediante microscopía electrónica de barrido, fue realizado en muestras representativas para los 3 campus de la Unach (Figura 4). Los valores indican la presencia de 11 elementos característicos y recurrentes en las muestras recolectadas. La composición elemental de las muestras contiene partículas identificadas en menor proporción ( $\mathrm{Al}, \mathrm{Ca}, \mathrm{Na}, \mathrm{K}, \mathrm{Mg}$ y Ti), pero también existen partículas que varían considerablemente y comprende componentes elevados de $\mathrm{O}$, $\mathrm{Si}$, Fe y C.
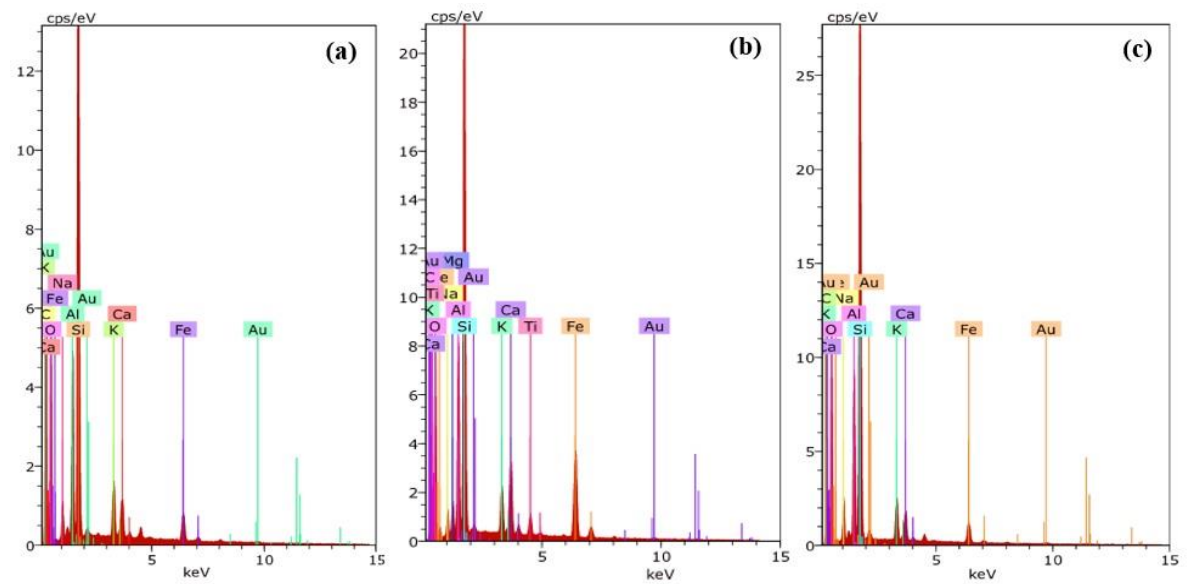

Figura 4: Análisis de composición molecular: (a) Campus Edison Riera; (b) Campus Centro; (c) Campus La Dolorosa.

El análisis cuantitativo de las diferentes morfologías de partículas PMS, confirman la presencia de varios elementos presentes en el aire como: $\mathrm{Al}, \mathrm{Na}, \mathrm{Ca}, \mathrm{Fe}$ y $\mathrm{K}$, encontrándose con mayor porcentaje al O, Si y C. La tabla 4 muestra la composición cuantitativa de elementos en diferentes partículas obtenidas en el papel filtro, en las tres estaciones de monitoreo RF1, RF2, y RF3. La presencia de C en las muestras analizadas, atribuye a que es un componente principal del PMS (polvos) y de combustibles fósiles provenientes principalmente del tráfico vehicular (Talbi et al., 2017). Así mismo 
este elemento puede concentrarse en el aire por varios días y semanas, para posteriormente precipitar al suelo (Manso \& Carrillo, 2018). Sin embargo, vale la pena indicar que la presencia de $\mathrm{Au}$, se debe al recubrimiento con este elemento realizado sobre la muestra como pretratamiento, con el objetivo de otorgar carácter conductor al material analizado (Ipohorski \& Bozzano, 2013).

Tabla 4: Composición química elemental de las muestras de PM. Los valores se expresan con porcentaje en peso.

\begin{tabular}{cccccc}
\hline \multicolumn{2}{c}{ Campus Edison Riera (RF1) } & \multicolumn{2}{c}{ Campus Centro (RF2) } & \multicolumn{2}{c}{ Campus La Dolorosa (RF3) } \\
\hline Elemento & p.p., $\%$ & Elemento & p.p., $\%$ & Elemento & p.p., \% \\
\hline $\mathrm{O}$ & 43.5 & $\mathrm{O}$ & 45.0 & $\mathrm{O}$ & 51.5 \\
$\mathrm{C}$ & 35.7 & $\mathrm{Si}$ & 21.1 & $\mathrm{Si}$ & 21.6 \\
$\mathrm{Si}$ & 11.2 & $\mathrm{Fe}$ & 8.2 & $\mathrm{C}$ & 10.6 \\
$\mathrm{Al}$ & 3.7 & $\mathrm{Al}$ & 7.6 & $\mathrm{Al}$ & 6.8 \\
$\mathrm{Na}$ & 1.7 & $\mathrm{C}$ & 7.4 & $\mathrm{Na}$ & 3.6 \\
$\mathrm{Fe}$ & 1.5 & $\mathrm{Ca}$ & 3.5 & $\mathrm{Fe}$ & 2.0 \\
$\mathrm{~K}$ & 1.1 & $\mathrm{Na}$ & 2.2 & $\mathrm{~K}$ & 1.8 \\
$\mathrm{Ca}$ & 1.0 & $\mathrm{~K}$ & 1.8 & $\mathrm{Ca}$ & 1.5 \\
$\mathrm{Au}$ & 0.5 & $\mathrm{Mg}$ & 1.6 & $\mathrm{Au}$ & 0.8 \\
- & - & $\mathrm{Ti}$ & 1.1 & - & - \\
- & - & $\mathrm{Au}$ & 0.6 & - & - \\
\hline
\end{tabular}

De igual manera el $\mathrm{Si}$, combinado con otros minerales como $\mathrm{Al}$, $\mathrm{Ti}$ y $\mathrm{Ca}$, son elementos que forman parte de la composición del suelo y que a su vez provienen del polvo de vías y de áreas de construcción. Este polvo al ser arrastrado por el viento queda suspendido en el aire por varios días el cual se convierte en un riesgo para las personas (Quijano et al., 2010). Estos resultados concuerdan con otros estudios relacionados con el transporte del viento y la contaminación del aire por polvo en ciudades europeas (Chin et al., 2007).

Los elementos tales como $\mathrm{Fe}, \mathrm{K}$ y Na, provienen de la combustión de vehículos, desgaste de ruedas y frenos (Machado et al., 2008). El PM emitido a la atmosfera en concentraciones mayores a los límites permisibles produce efectos nocivos en la salud de las personas, el cual causando daños a los pulmones ocasionando afecciones respiratorias, muertes prematuras, cáncer, entre otras (Oyarzún, 2010; Ubilla \& Yohannessen, 2017). Al comparar los metales hallados en los campus de la Unach observamos cierta similitud en cuanto a la presencia de: O, C, Si, Al y Fe. Los sitios en estudio presentan propiedades antropogénicas (tráfico vehicular, industria y construcción) y natural (partículas de polvo, producto de la caída de ceniza del volcán Tungurahua). La presencia de Fe puede provenir de la resuspensión del polvo al paso de automóviles y de su combustión en el caso de vehículos a diésel. También existe una pequeña contribución de actividades antropogénicas en esta área por estar rodeada de una gran densidad habitacional (el hierro producto de la actividad constructora y metalúrgica). Los niveles más altos del contenido de $\mathrm{C}$ en las estaciones de muestreo RF1 y RF3 está relacionado con el alto tráfico vehicular y su proceso de combustión incompleta. Por lo contrario, los niveles bajos de este elemento en la estación RF2 obedecen a la reducida área del campus universitario que impide la movilización vehicular.

\subsection{Caracterización física}

Las micrografías SEM de PMS, recolectadas en las estaciones de monitoreo de la Unach se muestran en la figura 5. Las micrografías revelan que las muestras analizadas se caracterizan por poseer 
partículas de forma irregular y de diferentes tamaños, predominando. De acuerdo a su diámetro, las partículas dominantes corresponden a la fracción de menos de $10 \mu \mathrm{m}$.

Las micrografías muestran que estas partículas tienen forma de prismas rectangulares con predomino de ancho de 4 a $5 \mu \mathrm{m}$ (Figura 5). Se puede observar dos tipos de partículas según su morfología: (a) naturales; partículas de polvo (minerales) que tienen superficies rugosas, a veces forman agregados estructurales con formas y tamaños irregulares; (b) antropogénicas; su morfología por lo general es de forma esférica y redondeada con una superficie lisa, lo que revela combustión u otro proceso desarrollado a alta temperatura (Radulescu et al., 2017).

El análisis de las diferentes micrografías confirma la presencia de varios elementos como: $\mathrm{O}, \mathrm{Si}, \mathrm{Fe}$, $\mathrm{Al}, \mathrm{C}, \mathrm{Ca}, \mathrm{Na}, \mathrm{K}, \mathrm{Mg}$, Ti; que se originan a partir de fuentes naturales y antropogénicas (por ejemplo, $\mathrm{SiO}_{2}, \mathrm{CaCO}_{3}$ y $\mathrm{CaSO}_{4}$, este último producido por la reacción química entre $\mathrm{CaCO}_{3}$ y $\mathrm{SO}_{2}$ durante la combustión de combustibles). Los sulfatos son derivados del combustible diésel y en pequeñas cantidades de aceites lubricantes. También se forman por la oxidación del dióxido de azufre $\left(\mathrm{SO}_{2}\right)$ emitido por los procesos de combustión, ya que, por los alrededores de los campus de la Unach, no se localizan industrias de gran magnitud que puedan aportar con partículas a la atmosfera; esto se ve reflejado por la cantidad de vehículos que entran y salen de los campus universitarios.

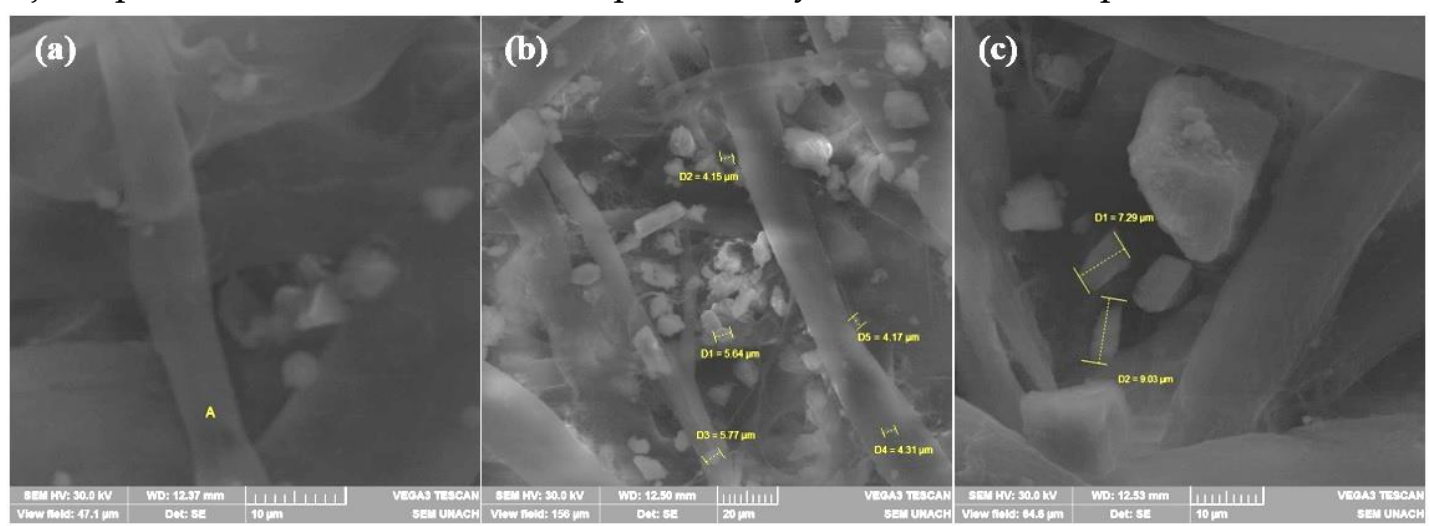

Figura 5: Micrografías SEM de material particulado sedimentable: (a) Campus Edison Riera; (b) Campus Centro y (c) Campus La Dolorosa.

\section{Modelo geoestadístico}

La distribución espacial de $\mathrm{PM}_{2.5}$ predicho por el método kriging, para el campus Edison Riera, muestra un patrón o tendencia espacial similar. El mapa resultante del modelo muestra cinco áreas con diferentes distribuciones espaciales que van desde los $0.28 \mu \mathrm{g} / \mathrm{m}^{3}$ hasta $31.0 \mu \mathrm{g} / \mathrm{m}^{3}$ y con una dirección del viento en sentido norte a sur (Figura 6).

El primer valor está representado por un área mínima distribuida en la parte centro y norte de la zona de estudio. En esta área, la concentración media de $\mathrm{PM}_{2.5}$ fue de $1.29 \mu \mathrm{g} / \mathrm{m}^{3}$ con un rango que va desde 0.28 a $2.3 \mu \mathrm{g} / \mathrm{m}^{3}$. La segunda y más grande está representada por una diagonal que va de norte a sur cubriendo la mayor área de la zona de estudio. La concentración media de $\mathrm{PM}_{2.5}$ normalizado fue de aproximadamente $4.35 \mu \mathrm{g} / \mathrm{m}^{3}$ con un rango de 2.3 a $6.4 \mu \mathrm{g} / \mathrm{m}^{3}$. La tercera zona presenta un valor medio de $9.7 \mu \mathrm{g} / \mathrm{m}^{3}$ con un rango que va de $6.4 \mu \mathrm{g} / \mathrm{m}^{3}$ a $13.0 \mu \mathrm{g} / \mathrm{m}^{3}$ y se ubica en la parte norte del campus Edison Riera. La cuarta escala de distribución cubre las áreas del centro y norte, más la parte sur del campus Edison Riera. La concentración media de PM2.5 normalizada fue de $17.0 \mu \mathrm{g} / \mathrm{m}^{3}$ con un rango intercuartílico entre $13 \mu \mathrm{g} / \mathrm{m}^{3}$ y $21 \mu \mathrm{g} / \mathrm{m}^{3}$.

La quinta y última área está en la parte centro y sur del campus; donde la concentración media fue de $26 \mu \mathrm{g} / \mathrm{m}^{3}$ con un rango que va desde $21 \mu \mathrm{g} / \mathrm{m}^{3}$ a $31 \mu \mathrm{g} / \mathrm{m}^{3}$. Esta área mostro las mayores concentraciones de $\mathrm{PM}_{2.5}$ normalizado. Finalmente, es importante resaltar que los valores altos están 
relacionados con la frecuente concurrencia de vehículos los cuales realizan emanaciones producto de la combustión incompleta de combustibles fósiles. Por lo contario la menor concentración de $\mathrm{PM}_{2.5}$ se encontró principalmente en la zona central en donde se exhibieron valores por debajo de $2.4 \mu \mathrm{g} / \mathrm{m}^{3}$.

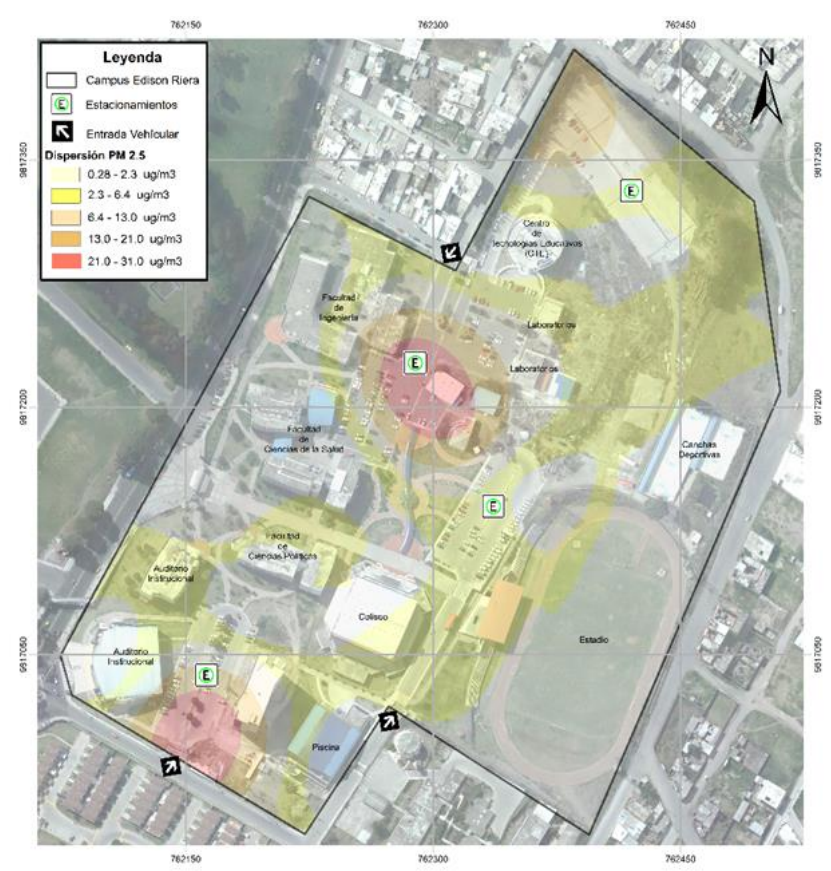

Figura 6: Dispersión Material Particulado PM2.5 Campus Edison Riera.

El análisis geoestadístico de PM2.5 en el campus Centro (Figura 7), demostró que los niveles promedio más altos se dieron en el área de estacionamiento $\left(4.7-6.4 \mu \mathrm{g} / \mathrm{m}^{3}\right)$. Esta franja abarca las horas pico del tráfico en el campus universitario, es decir que en estas horas se presentan los mayores niveles de desplazamiento vehicular.

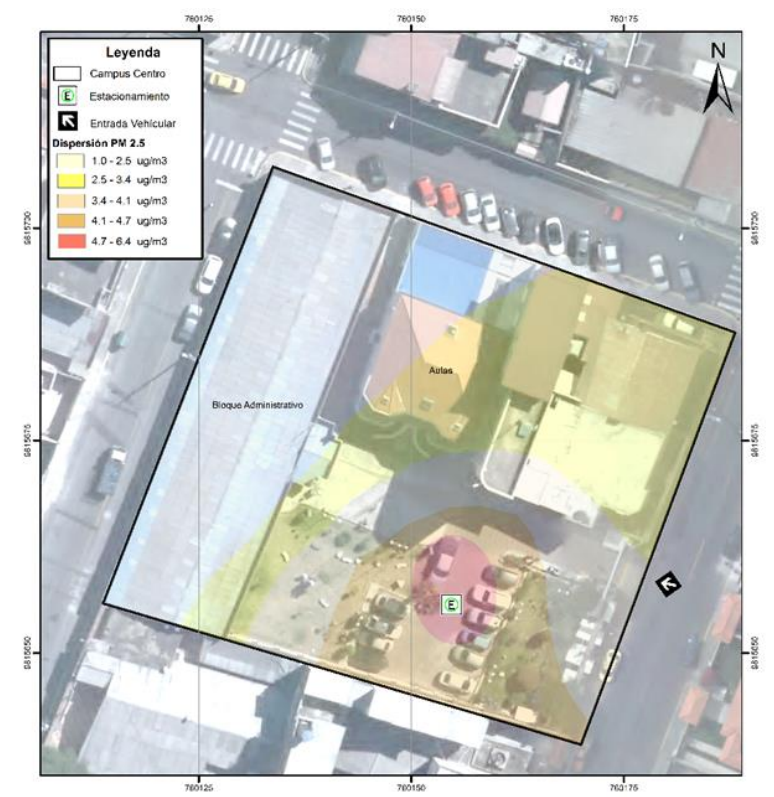

Figura 7: Dispersión Material Particulado PM 2.5 campus Centro.

La Figura 8, muestra la distribución espacial de PM 2.5 en el campus La Dolorosa, en donde se puede observar la presencia de 3 zonas, que presentan valores por encima de $10.0 \mu \mathrm{g} / \mathrm{m}^{3}$. Estos valores están relacionados con zonas de entrada de vehículos y áreas de estacionamientos; lo cual indica una relación directa con el parque automotor que ingresa a la institución. Los resultados de 
distribución espacial de $\mathrm{PM}_{2.5}$ estimados con la interpolación kriging, fueron comparados con la legislación ecuatoriana MAE (2017), en donde se establece como límite máximo permisible de PM2.5 el valor de $65 \mu \mathrm{g} / \mathrm{m}^{3}$; así como también con el límite internacional de $25 \mu \mathrm{g} / \mathrm{m}^{3}$ propuesto por la OMS (2005). Esta comparación indicó que los valores de distribución espacial obtenidos para el campus no sobrepasan los límites máximos permisibles, por lo que no son considerados como nocivos para la salud.

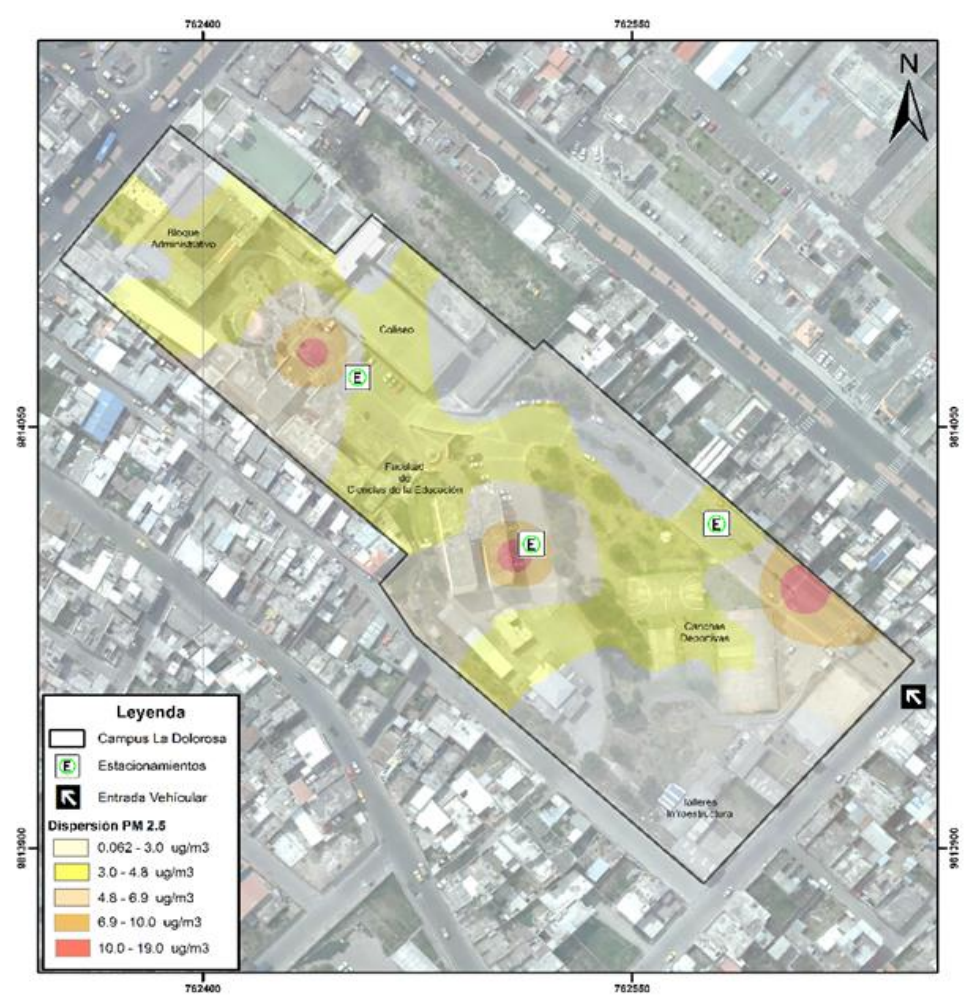

Figura 8: Dispersión Material Particulado PM 2.5 campus La Dolorosa.

\section{Discusión}

Los resultados obtenidos del monitoreo de PM, indican que los valores están dentro de los límites máximos permisibles establecidos por la legislación ecuatoriana $\left(65 \mu \mathrm{g} / \mathrm{m}^{3}\right)$ y por la OMS $(25$ $\left.\mu \mathrm{g} / \mathrm{m}^{3}\right)$. Es importante indicar también que existen valores que están muy cercanos a los límites establecidos por la OMS y por ello resulta importante realizar posteriores estudios que permitan descubrir al PM, como un predictor de los efectos sobre la salud de las personas. La presencia de valores bajos de PM2.5 y PM10, obedecen a lo descrito por Hernández et al., (2013), quienes mencionan que en sectores donde se levantó la información presentan suelos pavimentados y escasos en polvaredas.

De igual forma nuestros hallazgos coinciden con lo descrito por Hinojosa et al., (2019), en un estudio realizado en la ciudad de México a partir de 37 exposiciones personales. Por lo contrario, en la estación RF1 se logró identificar uno de los valores medios más altos $\left(10.82 \mu \mathrm{g} / \mathrm{m}^{3}\right)$, lo que indica que existe contaminación a causa de estas partículas por lo que son motivo de preocupación debido a su alta dimensión, así como a sus características fisicoquímicas. Por ello el tamaño de partículas juega un papel preponderante al momento de su ingreso en las vías respiratorias; por lo que se menciona, que a medida de que su tamaño se reduce mayor es su capacidad de penetrar directamente en las vías respiratorias y ocasionar alteraciones en este sistema (Candanoza et al., 2013; Oyarzún, 2010). Así mismo se ha demostrado que la morfología esférica de las partículas está asociada con los niveles de hierro, que se originan a partir de fuentes naturales y antropogénicas, lo que les convierten en un factor de riesgo asociado a la salud de las personas (Radulescu et al., 2017). 
De hecho al clasificar los valores de los promedios diarios y anuales sugeridos en las guías de la OMS, se estableció que el promedio de las 24 horas no se excedió la concentración de dichas guías de $25 \mu \mathrm{g} / \mathrm{m}^{3}$ (Rodríguez et al., 2020). Sin embargo, se sabe que una de las mayores fuentes de PM es la combustión que proviene de los vehículos que ingresan y salen de los campus universitarios. En base a lo anterior las estaciones de monitoreo fueron ubicadas estratégicamente en los sitios de acceso principal de la zona de estudio donde permitió registrar gran afluencia vehicular, la cual contribuye considerablemente en las emisiones de PM.

Para identificar el efecto causal del PMS, se realizó un análisis de RDX que muestran la presencia de elementos metálicos los cuales presentan resultados que concuerdan con otros estudios realizados en áreas urbanas en donde se demuestra que el polvo y las partículas presentes en la vías están relacionados con el tráfico vehicular, la quema de combustible y otros procesos industriales (Talbi et al., 2017). La composición elemental de PMS realizado a las muestras indican que cerca del $28 \%$ de los elementos presenta valores superiores a 10 respecto al rango de su peso y alrededor del $72 \%$ muestran valores por debajo de 8 .

En cuanto al análisis de la distribución espacial de $\mathrm{PM}_{2,5}$ se ha demostrado que la contaminación está relacionada directamente con los vehículos automotores como fuente de emisión. Por esta razón el presente estudio ha intentado demostrar la ocurrencia de la contaminación del aire mediante el uso del modelo de interpolación Kriging. Este modelo se puede utilizar como un enfoque alternativo a un modelo de dispersión que permite obtener una relación estadística entre las características de sector y las concentraciones de $\mathrm{PM}_{2,5}$ medidas mediante un equipo móvil y en un número determinado de sitios. Con ello se pudo predecir la concentración de $\mathrm{PM}_{2,5}$ en los campus de la Unach, donde se observaron valores que van desde $0.28 \mu \mathrm{g} / \mathrm{m}^{3}$ hasta $31.0 \mu \mathrm{g} / \mathrm{m}^{3}$. En las figuras se observa un diagnóstico general del comportamiento de $\mathrm{PM}_{2,5}$ en donde la coloración rojiza indica áreas de mayor concentración; sin embrago no quiere decir que su valor está por encima de los límites permisibles estipulados en la legislación ecuatoriana MAE (2017) y en lo propuesto por la OMS (2005).

En general, del presente estudio podemos concluir que la contaminación causada por partículas finas (PM) es motivo de preocupación debido a su gran dimensión y sus características fisicoquímicas, por lo que los datos generados son una herramienta importante que puede ser utilizada para ayudar a las autoridades de la institución y a los tomadores de decisiones a definir planes locales para la gestión de la calidad del aire y aumentar la conciencia de la población.

\section{Conclusiones}

La evaluación de PMS en el área de estudio no supera la legislación ecuatoriana $\left(65 \mu \mathrm{g} / \mathrm{m}^{3}\right)$, pero muestra una tendencia muy cercana a los límites establecidos, en especial a lo que recomienda la OMS. Esto determina la necesidad de seguir con los monitoreos; así mismo, que se realice la difusión de la información obtenida, para concientizar a la población universitaria incluidas sus autoridades ante los riesgos de exposición en especial en los días que se presentan emisiones de polvo producto de la fuerza de las ruedas de los vehículos y corrientes fuertes de aire.

La caracterización morfológica de las partículas de PM, mostro formas de tipo esférica, redondeada, irregular, placas angulares y porosas las cuales indican combustión incompleta $\mathrm{u}$ otro proceso realizado a altas temperaturas. La presencia de partículas finas menores a $10 \mu \mathrm{m}$, tiene su explicación en el desarrollo de actividades antropogénicas (tráfico vehicular, industria y construcción) y naturales (partículas de polvo, producto de la caída de ceniza del volcán Tungurahua). Esta situación es preocupante debido a que estas partículas representan mayor riesgo para la salud, especialmente en la población más vulnerable. 
La composición química de $\mathrm{PM}_{2.5}$ proporciona datos relevantes con respecto a la evaluación de fuentes de emisión. El análisis SEM-EDS de las muestras de PM2.5 revela detalles importantes sobre el grupo orgánico e inorgánico de las partículas en el aire. Los peligros de los metales tóxicos para la salud humana son bien conocidos, considerando el riesgo carcinogénico de elementos como el Al y Fe.

Los mapas de distribución espacial de PM2.5 generados por software ArcGIS 10.x, permitieron definir que los radios críticos de concentración de $\mathrm{PM}_{2.5}$ se encuentran aproximadamente a 50 metros de los focos de emisión de contaminantes. Este radio de deposición tan pequeño, está condicionado por los mayores niveles de desplazamiento vehicular, vientos y sobre todo porque en las zonas no se produce emisiones de contaminantes en altura, sino que se producen las emisiones a pocos metros de la superficie. Con ello se demuestra que la modelación de PM a través de herramientas Spatial Analyst y Geostatistical Analyst, brinda resultados cercanos a la realidad y que puede convertirse en uno de los modelos más usados para estudiar el comportamiento de las partículas.

\section{Contribución de los autores}

En concordancia con la taxonomía establecida internacionalmente para la asignación de créditos a autores de artículos científicos (https://casrai.org/credit/). Los autores declaran sus contribuciones en la siguiente matriz:

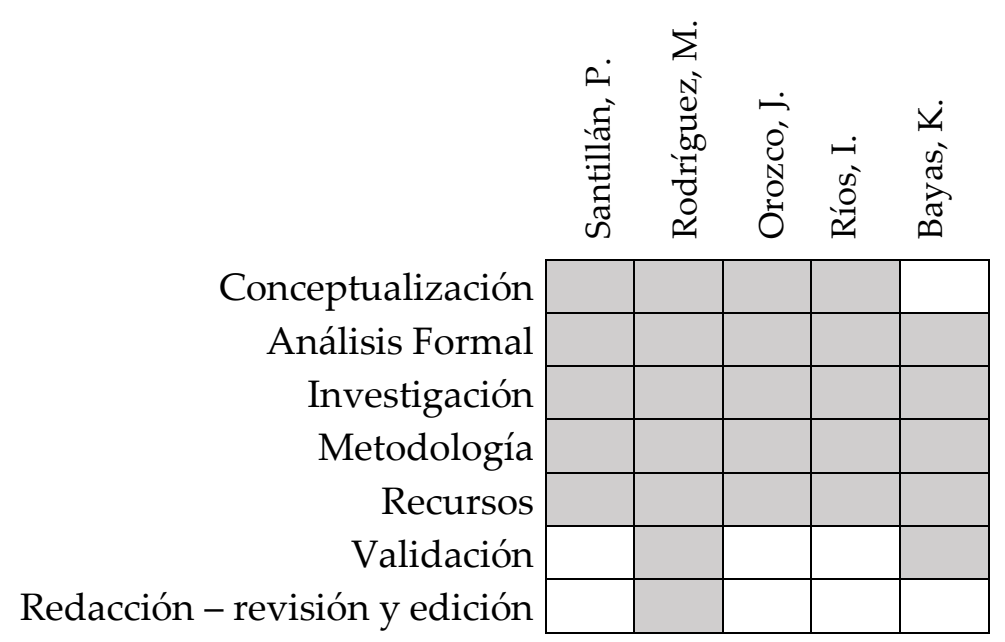

\section{Conflicto de Interés}

Los autores deben declarar que no existen conflictos de interés de naturaleza alguna o en su defecto declarar el tipo de conflicto de interés que el autor (o autores) mantenga con la presente investigación.

\section{Agradecimiento}

Los autores agradecen a la Universidad Nacional de Chimborazo por permitir desarrollar la presente investigación y al Laboratorio de Microscopía Electrónica de la Universidad Nacional de Chimborazo en la persona de su director el Dr. Víctor Julio García por haber tomado las micrografías y realizado el análisis EDX a las muestras. 


\section{Referencias}

Almirón, M., Dalmasso, A., \& Llera, J. (2008). Uso de Larrea cuneifolia Cav. y Zuccagnia punctata Cav. en la evaluación del material particulado sedimentable en una calera de Los Berros- San Juan-Argentina. Multequina, 17, 29-38. https://www.redalyc.org/pdf/428/42801704.pdf

Bayas, K. (2017). Distribución espacial y multitemporal de material particulado, en los campus universitarios de la Unach de la ciudad de Riobamba (Trabajo de investigación previo a la obtención del título de Ingeniero Ambiental), Universidad Nacional de Chimborazo, Ecuador. Recuperado de: http://dspace.unach.edu.ec/handle/51000/4117

Bustillos, J., Romero, J., Guevara, A., \& Díaz, J. (2018). Tephra fallout from the long-lasting Tungurahua eruptive cycle ( 1999-2014 ): Variations through eruptive style transition and deposition processes. Andean Geology, 45(1), 47-77. https://doi.org/10.5027/andgeoV45n13036

Canales, M., Quintero, M., Castro, T., \& García, R. (2014). Las partículas respirables PM10 y su composición química en la zona Urbana y rural de Mexicali, Baja California en México. Información Tecnológica, 25(6), 13-22. https://doi.org/10.4067/S0718-07642014000600003

Candanoza, S., Goribar, L., \& García, F. (2013). Relación partículas respirables (PM10)/ partículas suspendidas totales (PST) en Santa Marta (Colombia). Dyna, 80(179), 157-164. http://www.scielo.org.co/pdf/dyna/v80n179/v80n179a18.pdf

Celis, J., Morales, J., Zaror, C., \& Carvacho, O. (2007). Contaminación del aire atmosférico por material particulado en una ciudad intermedia: El caso de Chillán (Chile). Información Tecnológica, 18(3), 49-58. https://doi.org/10.4067/S0718-07642007000300007

Chin, M., Diehl, T., Ginoux, P., \& Malm, W. (2007). Intercontinental transport of pollution and dust aerosols: Implications for regional air quality. Atmospheric Chemistry and Physics, 7(21), 55015517. https://acp.copernicus.org/articles/7/5501/2007/acp-7-5501-2007.pdf

Few, R., Armijos, M. T., \& Barclay, J. (2017). Living with Volcan Tungurahua: The dynamics of vulnerability during prolonged volcanic activity. Geoforum, 80, 72-81. https://doi.org/10.1016/j.geoforum.2017.01.006

GADM Riobamba. (2020). Plan de Desarrollo y Ordenamiento Territorial del Cantón Riobamba. Recuperado de: http://www.gadmriobamba.gob.ec/index.php/descarga/category/1096-plande-desarrollo-y-ordenamiento-territorial-2020-2030

García, P., \& Rojas, N. (2016). Análisis del origen de PM 10 y PM 2.5 en Bogotá usando gráficos polares. Mutis, 6(2), 47-58. https://revistas.utadeo.edu.co/index.php/mutis/article/view/1150/1193

Hernández, J., Flores, J., Flores, M., Rodríguez, N., Delgado, M., Shukla, M., \& DuBois, D. (2013). Material particulado dispersado al aire en áreas sin asfalto en Ciudad Juárez. Revista de Ciencia y Tecnología de La UACJ, 11, 9-14. http://bva.colech.edu.mx/xmlui/handle/1/1638

Hinojosa, I., Infante, O., \& Vallejo, M. (2019). Distribution of PM2.5 air pollution in Mexico City: Spatial analysis with land-use regression model. Applied Sciences (Switzerland), 9(14), 1-16. https://doi.org/10.3390/app9142936

INAMHI. (2019). Anuario Climatológico 2019. Recuperado de: http://www.sushifeel.com/site/a1bfe1anuario-meteorol\%C3\%B3gico-inamhi-2019-pdf

INEC. (2010). Censo de Población y Vivienda 2010. http://www.ecuadorencifras.gob.ec/wp- 
content/descargas/Manu-lateral/Resultados-provinciales/chimborazo.pdf

Ipohorski, M., \& Bozzano, P. (2013). Microscopía electrónica de barrido en la caracterización de materiales. Ciencia e Investigación, 63(3), 43-53. Recuperado de : http://aargentinapciencias.org/wp-content/uploads/2018/01/RevistasCeI/tomo63-3/5MICROSCOPIA-ELECTRONICA-DE-BARRIDO-EN-LA-CARACTERIZACION-DEMATERIALES-cei63-3-2013-5.pdf

Machado, A., García, N., García, C., Acosta, L., Córdova, A., Linares, M., Giraldoth, D., \& Velásquez, H. (2008). Contaminación por metales ( $\mathrm{Pb}, \mathrm{Zn}, \mathrm{Ni}$ y $\mathrm{Cr}$ ) en aire, sedimentos viales y suelo en una zona de alto tráfico vehicular. Rev. Int. Contam. Ambient., 24(4), 171-182. http://www.scielo.org.mx/scielo.php?script=sci_arttext\&pid=S0188-49992008000400003

MAE. (2017). Texto Unificado de Legislación Secundaria de Medio Ambiente, TULSMA (Issue 3399). Recuperado de:

https://www.ambiente.gob.ec/wp-content/uploads/downloads/2018/05/TULSMA.pdf

Manso, R., \& Carrillo, E. (2018). Emisiones de dióxido de carbono equivalente, dioxinas y carbono negro en la región occidental de Cuba provocada por incendios forestales. Revista Cubana de Meteorología, 24, 405-411. http://rcm.insmet.cu/index.php/rcm/article/view/446/566

Minitab. (2016). Getting Started with Minitab 17. Minitab. In Reference manual (p. 88). https://www.minitab.com/uploadedFiles/Documents/gettingstarted/Minitab17_GettingStarted-en.pdf

OMS. (2005). Guías de calidad del aire de la OMS relativas al material particulado, el ozono, el dióxido de nitrógeno y el dióxido de azufre. Recuperado de:

https://www.who.int/phe/health_topics/AQG_spanish.pdf

Oyarzún, M. (2010). Contaminación aérea y sus efectos en la salud. Revista Chilena de Enfermedades Respiratorias, 26(1), 16-25. https://scielo.conicyt.cl/scielo.php?pid=S0717$73482010000100004 \&$ script $=$ sci_arttext\&tlng=en

Palacio, D. F., Zafra, C. A., \& Rodríguez, J. P. (2014). Evaluación de la calidad del aire mediante un laboratorio móvil: Puente Aranda (Bogotá D . C ., Colombia), Revista Facultad Ingenieriería Universidad Antioquía, (2), 153-166. Recuperado de

https://revistas.udea.edu.co/index.php/ingenieria/article/download/15069/16710

Quijano, A., Quijano, M., \& Henao, J. (2010). Caracterización fisicoquímica del material particuladofracción respirable PM2.5 en Pamplona-Norte de Santander-Colombia. Revista de La Facultad de Ciencias Básicas, 8(1), 1-20. https://www.redalyc.org/pdf/903/90315226007.pdf

Radulescu, C., Stihi, C., Iordache, S., Dunea, D., \& Dulama, I. (2017). Characterization of Urban Atmospheric PM2 . 5 by ATR-FTIR, ICP-MS and SEM-EDS Techniques. Rev. Chim, 68(4), 805-810. https://doi.org/10.37358/RC.17.4.5557

Rodríguez, L., Sierra, R., \& Blanco, L. (2020). Análisis espacial de las concentraciones de PM2,5 en Bogotá según los valores de las guías de la calidad del aire de la Organización Mundial de la Salud para enfermedades cardiopulmonares, 2014-2015. Biomédica, 40, 137-152. https://www.ncbi.nlm.nih.gov/pmc/articles/PMC7357390/

Salinas, P. V. (2012). Contaminación atmosférica por material particulado y consultas de urgencia por morbilidad respiratoria en menores de 5 años en la ciudad de Valdivia, periodo mayo-junio, año 2010 (Tesis presentada como parte de los requisitos para optar al grado de Licenciado en Enfermería), Universidad Austral de Chile, Chile. Recuperado de: 
http://cybertesis.uach.cl/tesis/uach/2012/fms165c/doc/fms165c.pdf

Santillán, G., Damián, D., Rodríguez, M., Torres, S., Cargua, F., \& Torres, S. (2016). Estimación del grado de contaminación de material particulado atmosférico y sedimentable en el laboratorio de servicios ambientales de la UNACH. Perfiles, 2, 32-39.

Scapini, V., Carrasco, C., \& Vergara, C. (2018). Efectos de la contaminacíon del aire en atenciones de urgencia de la Regíon Metropolitana. Revista Ingeniería de Sistemas, 32, 55-74. https://www.researchgate.net/profile/Cinthya_Vergara_Silva/publication/327537047.pdf

Talbi, A., Kerchich, Y., Kerbachi, R., \& Boughedaoui, M. (2017). Assessment of annual air pollution levels with PM1, PM2.5, PM10 and associated heavy metals in Algiers, Algeria. Environmental Pollution, 232, 1-12. https://doi.org/10.1016/j.envpol.2017.09.041

Ubilla, C., \& Yohannessen, K. (2017). Contaminación atmosférica efectos en la salud respiratoria en el niño. Revista Médica Clínica Las Condes, 28(1), 111-118. https://doi.org/10.1016/j.rmclc.2016.12.003 